\title{
IUFOST2006/1093 Designing foods for sensory pleasure
}

\author{
M. Kerslake \\ Numsight, 80/82 rue Gallieni, 92100 Boulogne-Billancourt, France \\ m.kerslake@numsight.com
}

The arrival of industrialised food brought with it an increased scientific understanding, inventions and great product development. As time passed it was realised that there needed to be ways of measuring and quantifying the hedonic or pleasurable aspects of food and the sensorial sciences developed slowly to meet this need.

In the 1970's and early 1980's when techniques were first being used on a wider scale, it was easy to find products with large disparities in consumer appreciation - these days it is much less likely. Every company has scientists working hard to formulate good products for consumers and every company, both large and small, is doing consumer research of one kind or another - and many companies can identify at the same time an idea that will satisfy Consumer expectations.

The challenge is no longer to spot the idea that will deliver business, but to be first to develop it and to deliver best fit of product to concept expectations. Food scientists often approach formulation in the same way; some science, a good dose of creativity, but mainly lots of intuition - 'the Art'.

Today's science has the capability to help design better Art! Through product examples to be discussed, the key to exploiting the product opportunities we have, is identifying the levers for the formulation that turn a consumer on and off. It is no longer enough to be satisfied by the current way products are developed. It is necessary to re-think preference mapping approaches, deploy more experimental designs and discover how these evolving techniques can be used to design foods to deliver optimised consumer preference. 